\title{
Feasibility of automated proton therapy plan adaptation for head and neck tumors using cone beam CT images
}

Christopher Kurz ${ }^{1,2}$, Reinoud Nijhuis ${ }^{1}$, Michael Reiner ${ }^{1}$, Ute Ganswindt ${ }^{1}$, Christian Thieke ${ }^{1}$, Claus Belka', Katia Parodi ${ }^{2}$ and Guillaume Landry ${ }^{2, *^{*}}$

\begin{abstract}
Background: Intensity modulated proton therapy (IMPT) of head and neck (H\&N) tumors may benefit from plan adaptation to correct for the dose perturbations caused by weight loss and tumor volume changes observed in these patients. As cone beam $C T$ (CBCT) is increasingly considered in proton therapy, it may be possible to use available CBCT images following intensity correction for plan adaptation. This is the first study exploring IMPT plan adaptation on $\mathrm{CBCT}$ images corrected and delineated by deformable image registration of the planning $\mathrm{CT}(\mathrm{pCT})$ to the $\mathrm{CBCT}$, yielding a virtual $\mathrm{CT}(\mathrm{VCT})$.

Methods: A Morphons algorithm was used to deform the pCTs and corresponding delineations of 9 H\&N cancer patients to a weekly CBCT acquired within \pm 3 days of a control replanning $C T$ scan (rpCT). The IMPT treatment plans were adapted using the $\mathrm{VCT}$ and the adapted and original plans were recalculated on the rpCT for dose/ volume parameter evaluation of the impact of adaptation.

Results: On the $\operatorname{rpCT}$, the adapted plans were equivalent to the original plans in terms of target volumes $D_{95}$ and $V_{95}$, but showed a significant reduction of $D_{2}$ in these volumes. OAR doses were mostly equivalent or reduced. In particular, the adapted plans did not reduce parotid gland $D_{\text {mean, }}$ but the dose to the optical system. For three patients the spinal cord or brain stem received higher, though well below tolerance, maximum dose. Subsequent tightening of the treatment planning constraints for these OARs on new vCT-adapted plans did not degrade target coverage and yielded pCT equivalent plans on the vCT.

Conclusions: An offline automated procedure to generate an adapted IMPT plan on CBCT images was developed and investigated. When evaluating the adapted plan on a control rpCT we observed reduced $D_{2}$ in target volumes as major improvement. OAR sparing was only partially improved by the procedure. Despite potential limitations in the accuracy of the vCT approach, an improved quality of the adapted plans could be achieved.
\end{abstract}

Keywords: Intensity modulated proton therapy, Cone beam CT, Adaptive radiation therapy, Deformable image registration, Head and neck cancer

\footnotetext{
*Correspondence: G.landry@Imu.de

${ }^{2}$ Department of Medical Physics, Faculty of Physics,

Ludwig-Maximilians-Universität München, Munich, Germany

'Department of Radiation Oncology, LMU Munich, Munich, Germany
}

(c) 2016 Kurz et al. Open Access This article is distributed under the terms of the Creative Commons Attribution 4.0 International License (http://creativecommons.org/licenses/by/4.0/), which permits unrestricted use, distribution, and reproduction in any medium, provided you give appropriate credit to the original author(s) and the source, provide a link to the Creative Commons license, and indicate if changes were made. The Creative Commons Public Domain Dedication waiver (http://creativecommons.org/publicdomain/zero/1.0/) applies to the data made available in this article, unless otherwise stated. 


\section{Background}

Intensity modulated radiation therapy (IMRT) with photons can be considered current state-of-the-art for the treatment of head and neck $(\mathrm{H} \& \mathrm{~N})$ cancer [1-3]. However, intensity modulated proton therapy (IMPT) using spot scanning has been shown to yield superior dose distributions compared to IMRT for the treatment of H\&N cancer patients [4-8]. Still, plan adaptation may be desirable to optimally treat patients undergoing weight loss or other anatomical modifications during the radiation course [9-11]. To enable adaptive radiation therapy (ART), a time point for re-planning needs to be chosen and a new delineated diagnostic quality computed tomography $(\mathrm{CT})$ image of the patient is required. The time point may be fixed for all patients based on clinical experience, or a patient specific action level may be derived based on e.g., evaluation of the dose of the day. It is thus obvious that access to frequent, delineated diagnostic CT quality images is of high importance in an adaptive workflow.

In-room cone beam CT (CBCT) imaging, which is increasingly considered for proton therapy [12], provides information related to patient positioning as well as anatomical variations. However, direct dose calculation on CBCT images is not recommended for IMPT [13] due to their poor image quality $[14,15]$. Previous work [16-21] suggests that it is feasible to apply deformable image registration (DIR) to the original delineated planning CT (pCT) image to match the anatomy observed on CBCT images. This method of CBCT intensity correction yields an image, referred to as virtual CT (vCT) in this work, suitable to IMPT dose calculation [22-26] and automatically yields updated contours from the DIR [18, 23, 27]. A previous evaluation in terms of matching features and Dice coefficient showed good agreement between vCT contours and those from a physician [23].

The availability of a delineated CT image fulfills the basic technical requirements for adaptation of the IMPT plan. Furthermore, the automated nature of the vCT generation and delineation may allow for plan adaptation with little increase to the clinical workload. To the best of our knowledge there is no study in the literature reporting on plan adaptation for IMPT based on CBCT images. In this study we investigated the re-optimization of the initial IMPT plan using the automatically delineated vCT to restore the initial optimization objectives. We investigated the impact of this correction by recalculating the dose distributions from the original and updated plans on a delineated control replanning CT (rpCT) image taken within 3 days of the CBCT image used to generate the $\mathrm{vCT}$ by evaluating dose/volume histogram (DVH) parameters.

\section{Methods}

Clinical datasets of $9 \mathrm{H} \& \mathrm{~N}$ cancer patients undergoing IMRT were employed in this study. The patient data acquisition and anonymization protocol used in this study received approval from the ethics committee of the University Clinic of the Ludwig-Maximilians-Universität München (LMU Munich). 6 patients had lesions of the larynx or pharynx (Pat1-6) while 3 cases had nasal cavity lesions (Pat7-9) as detailed in Table 1. Dose prescriptions in this study follow the original clinical IMRT treatments. The sizes of the target volumes can be found in Additional file 1: Table S1. Each patient had a delineated pCT, a $\mathrm{CBCT}$ and delineated rpCT from the same physician. The pCT and rpCT images were acquired with a Toshiba Aquilion LB scanner and reconstructed on a $1.074 \mathrm{~mm} \times$ $1.074 \mathrm{~mm} \times 3 \mathrm{~mm}$ grid. The CBCT images were acquired with the on-board Elekta Synergy Linac imager equipped with XVI R4.5 and reconstructed on a $1 \mathrm{~mm} \times 1 \mathrm{~mm} \times$ $1 \mathrm{~mm}$ grid. The scan parameters were the same as in Landry et al. [23] IMPT plans were generated with a research version of a commercial treatment planning system (TPS) (RayStation 4.6, RaySearch Laboratories, Stockholm, Sweden) following the approach outlined in Kurz et al. [13]: A four field arrangement has been used for Pat1- 6 with larynx or pharynx lesions $\left(45^{\circ}, 90^{\circ}, 270^{\circ}\right.$ and $315^{\circ}$ on the

Table 1 Patient characteristics. $\Delta \mathrm{t}_{\mathrm{rp} C \mathrm{~T}}$ and $\Delta \mathrm{t}_{\mathrm{CBCT}}$ are the times between planning and replanning $\mathrm{CT}$ acquisition and between planning $C T$ and $C B C T$ acquisition respectively

\begin{tabular}{|c|c|c|c|c|c|c|c|c|}
\hline Patient identifier & Age & Sex & Tumor site & TNM stage & $\Delta \mathrm{t}_{\mathrm{rp} \subset \mathrm{T}}$ (days) & $\Delta \mathrm{t}_{\mathrm{CBCT}}$ (days) & $\begin{array}{l}\text { SIB prescription low } \\
\text { dose/high dose (Gy) }\end{array}$ & Number of SIB fractions \\
\hline Pat1 & 65 & M & Larynx & pT2pNOMO & 51 & 50 & $50 /-$ & 25 \\
\hline Pat2 & 54 & $\mathrm{~F}$ & Hypopharynx, esophagus & $\mathrm{cT} 4 \mathrm{CN} 2 \mathrm{MO}$ & 39 & 41 & $50.4 / 56$ & 28 \\
\hline Pat3 & 71 & M & Larynx & pT1bNOMO & 34 & 35 & $54 / 60$ & 30 \\
\hline Pat4 & 87 & M & Hypopharynx & $\mathrm{cT} 2 \mathrm{cN} 2 \mathrm{bM} 0$ & 33 & 34 & $54 / 60$ & 30 \\
\hline Pat5 & 49 & M & Nasopharynx & $\mathrm{cT} 2 \mathrm{cN} 2 \mathrm{bM} 0$ & 40 & 40 & $54 / 60$ & 30 \\
\hline Pat6 & 42 & M & Larynx & pT2bpN1M0 & 44 & 41 & $50.4 / 56$ & 28 \\
\hline Pat7 & 66 & M & Right paranasal sinus & $\mathrm{pT} 2 \mathrm{cNOMO}$ & 31 & 30 & $50.4 / 61.6$ & 28 \\
\hline Pat8 & 67 & M & Left paranasal sinus & pT3N2bM0 & 30 & 30 & $54.4 / 64$ & 32 \\
\hline Pat9 & 76 & M & Nasal cavity & сT3NOMO & 37 & 38 & $50.4 / 56$ & 28 \\
\hline
\end{tabular}


International Electrotechnical Commission (IEC) scale, with $90^{\circ}$ and $270^{\circ}$ blocked in the shoulder area), and a 3 field arrangement for Pat7-9 with nasal cavity lesions $\left(0^{\circ}\right.$, $100^{\circ}, 260^{\circ}$ on the IEC scale, with $0^{\circ}$ blocked in the nasal/ buccal area). For eight patients simultaneous integrated boost (SIB) plans using two dose levels (reported in Table 1) were generated, while for one patient only a single dose level was used, following again the dose prescriptions of the original clinical IMRT plans. Target definition was also adopted from clinical IMRT planning procedures. The high dose CTV has been retrieved from expansion of the GTV by a $5-7 \mathrm{~mm}$ margin, for high dose PTV generation an additional 5-7 mm margin was applied. The low dose CTV also covered the lymph node areas following the delineation approach of Grégoire et al. [28] It was extended by 5$7 \mathrm{~mm}$ to the low dose PTV. All treatment fields have been optimized simultaneously (multi-field optimization). For Pat1-6, the main PTV coverage limitation was the mean dose to the parotid glands, since the spinal cord and brain stem were easily spared by IMPT. For Pat7-9 the mean dose to the eye lens and the maximum dose to the optical nerves and chiasm were also critical. Planning risk volumes (PRV) were used for optimization for the spinal cord, brain stem, optical nerves and chiasm. Table 2 reports the TPSspecific DVH objectives employed for treatment plan optimization in this study. Treatment plans were accepted when exceeding a $V_{95}$ of $95 \%$ in the PTVs, except from two cases where a slightly lower $V_{95}(94 \%)$ was accepted due to OAR constraints.

vCT images were generated for each $\mathrm{CBCT}$ by performing DIR of the aligned $\mathrm{pCT}$ to the $\mathrm{CBCT}$ using a Morphons algorithm [29], which is image intensity independent. The tools described in Landry et al. were used with slight modifications to the procedure. In Landry et al. a translational registration was used for aligning the pCT and CBCT prior to DIR [23], while in this work we also allowed for small rotations (always below $5^{\circ}$ in this study) to simulate modern 6-degrees-of-freedom patient position correction. The rigid registration was performed on a region of interest (ROI) containing the spine and ranging from the $1^{\text {st }}$ to the $6^{\text {th }}$ vertebrae for Pat 1 to Pat6 and on the skull for Pat7 to Pat9. For evaluation, the rpCT was also rigidly registered to the $\mathrm{pCT}$ using the same ROIs. Manual tuning of the final registrations yielded corrections smaller than $1 \mathrm{~mm}$, confirming the quality of the rigid registrations. The cavity correction method described in Landry et al., where empty/full cavities on the CBCT take the CT numbers of air/water on the vCT, was used for all cases [23].

The registered $\mathrm{rpCT}$ and $\mathrm{vCT}$ were imported in the TPS with their respective contours and the dose distribution corresponding to the initial plan, optimized on basis of the pCT, was calculated on the rpCT. A new plan was designed on the vCT using the same optimization parameters as the initial plan and the DIRbased contours. The rpCT dose distribution corresponding to this adapted plan was compared to the one from the original plan using the DVH parameters listed in Table 2 and the corresponding rpCT contours.

The median and interquartile range of the distribution of the differences between the parameters from Table 2 evaluated for the $\mathrm{pCT}$ and $\mathrm{rpCT}$ (with and without vCTbased plan adaptation) was computed for the 9 patients. DVH parameters with and without plan adaptation for the rpCT were also compared by a paired Wilcoxon signed-rank test in order to find statistically significant differences caused by plan adaptation on basis of the $\mathrm{vCT}$.

\section{Results}

Figure 1 shows IMPT dose distributions from the original plan computed on the pCT and rpCT as well as the vCTadapted plan on the rpCT (labelled $\mathrm{rpCT}_{\text {adapt }}$ ) and on the vCT (labelled $\mathrm{vCT}_{\text {adapt }}$ ) for two representative patients.

Table 2 DVH parameters used in this study. Planning risk volumes were used for the brain stem, spinal cord, optic nerves and optic chiasm. D prescr. is the prescription dose. The TPS-specific DVH objectives used in treatment plan optimization are also presented

\begin{tabular}{lll}
\hline Organ at risk or target & DVH parameters evaluated & DVH objectives used for planning \\
\hline High dose PTV & $D_{95}, D_{2}, V_{95}$ & $V_{95}=100 \%, D_{\min }=100 \% \cdot D_{\text {prescr. }} D_{\text {max }}<105 \% \cdot D_{\text {prescr. }}$ \\
High dose CTV & $D_{95}, D_{2}$ & \\
Low dose PTV & $D_{95}, D_{2}, V_{95}$ & $V_{95}=100 \%, D_{\min }=100 \% \cdot D_{\text {prescr, }} D_{\max }<105 \% \cdot D_{\text {prescr. }}$ \\
Low dose CTV & $D_{95}, D_{2}$ & \\
Parotid glands & $D_{\text {mean }}$ & $D_{\operatorname{mean}}<26 \mathrm{~Gy}$ \\
Spinal cord & $D_{2}$ & $D_{\max }<53 \mathrm{~Gy}$ \\
Brain stem & $D_{2}$ & $D_{\max }<53 \mathrm{~Gy}$ \\
Optical nerves & $D_{2}$ & $D_{\max }<54 \mathrm{~Gy}$ \\
Optical chiasm & $D_{2}$ & $D_{\max }<56 \mathrm{~Gy}$ \\
Eye & $D_{2}$ & $D_{\max }<45 \mathrm{~Gy}$ \\
Eye lens & $D_{\text {mean }}$ & $D_{\operatorname{mean}}<10 \mathrm{~Gy}$ \\
\hline
\end{tabular}




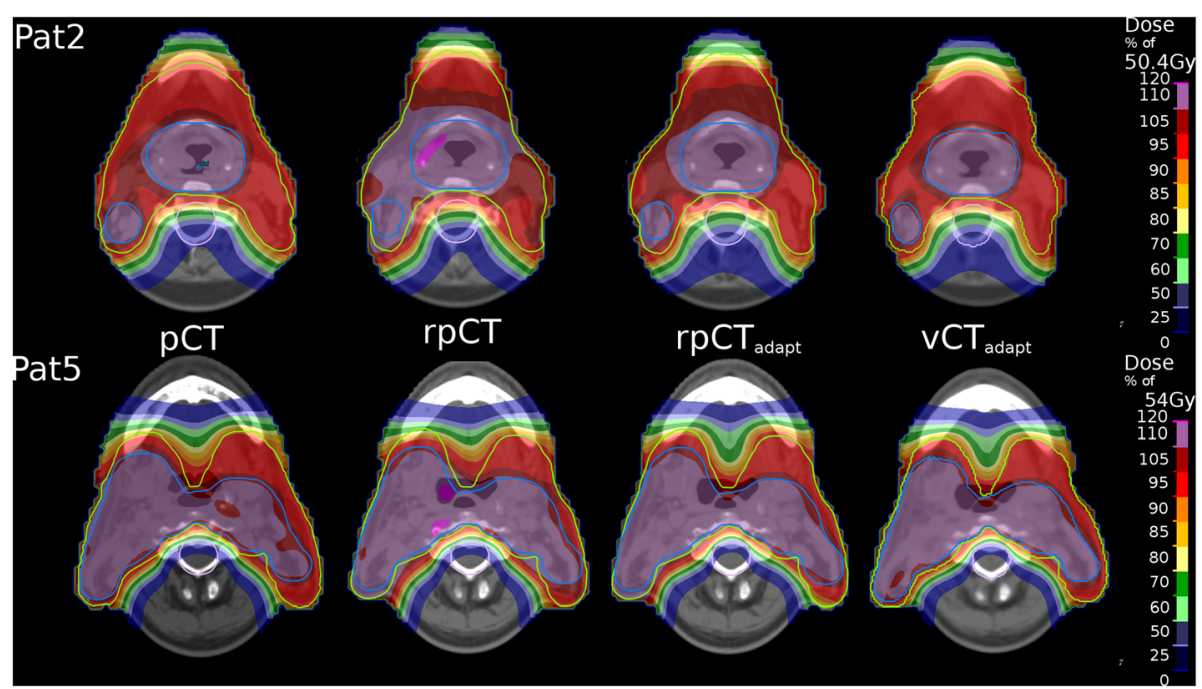

Fig. 1 Dose distributions for Pat2 and Pat5 from the original plan on the $\mathrm{pCT}$ and $\mathrm{rpCT}$ as well as the adapted dose distributions on the rpCT $(\mathrm{rpCT}$ adapt $)$ and $\mathrm{vCT}\left(\mathrm{vCT}_{\text {adapt }}\right)$. The high and low dose PTVs are indicated in blue and green respectively. The spinal cord PRV is also shown. For the high dose PTV, the $95 \%$ dose level corresponds to $105 \%$ on the color bar, which is relative to the low dose PTV prescription. Hot spots correspond to $120 \%$ or more of the low dose PTV prescription

Over-dosed regions in the low dose PTV were observed on the rpCT in the vicinity of the high dose PTV and the skin when applying the original plan for Pat2. This overdosage was reduced by adapting the plan using the vCT. For Pat 5 over-dosage was observed (up to $109 \%$ of the high dose PTV prescription dose, which corresponds to about $120 \%$ of the low dose PTV prescription dose) in the high dose PTV and could be eliminated in the adapted plan. In both cases, dose distributions of the adapted plan on the $\mathrm{vCT}$ were found similar to the original planning scenario $(\mathrm{pCT})$.

Figure 2 presents DVH curves for the original plan on the pCT and rpCT as well as the adapted plan on the rpCT and the vCT for the same two patients presented in Fig. 1. For both patients, over-dosed regions were observed in the low dose and high dose PTVs for the original plan on the rpCT. For Pat5, the target coverage of the original plan for the low dose PTV was degraded on the $\operatorname{rpCT}\left(D_{95}\right.$ reduction of $\left.4 \mathrm{~Gy}\right)$; for the adapted plan, coverage was almost recovered. The adapted plans mitigated over-dosage (above $105 \%$ of prescription) for both targets and both patients. The spinal cord $D_{2}$ was similar for the pCT, rpCT and rpCT with adapted plan for Pat5, while for Pat2 it rose from 42 Gy to 47 Gy and 45 Gy respectively. The adapted plan did not reduce the spared parotid gland $D_{\text {mean }}$ for both patients. In both cases, the adapted plan on the vCT exhibits similar DVH curves as the original plan on the $\mathrm{pCT}$.

Figures 3 and 4 present box plots of the distribution of differences of DVH indices for the target volumes of all patients. Absolute differences of the original and adapted plan on the rpCT with respect to the original dose distribution on the pCT are shown (top row in Fig. 3, left in Fig. 4), as well as DVH index differences between original and adapted plan on the rpCT (bottom row in Fig. 3, right in Fig. 4). The Wilcoxon rank-sum test
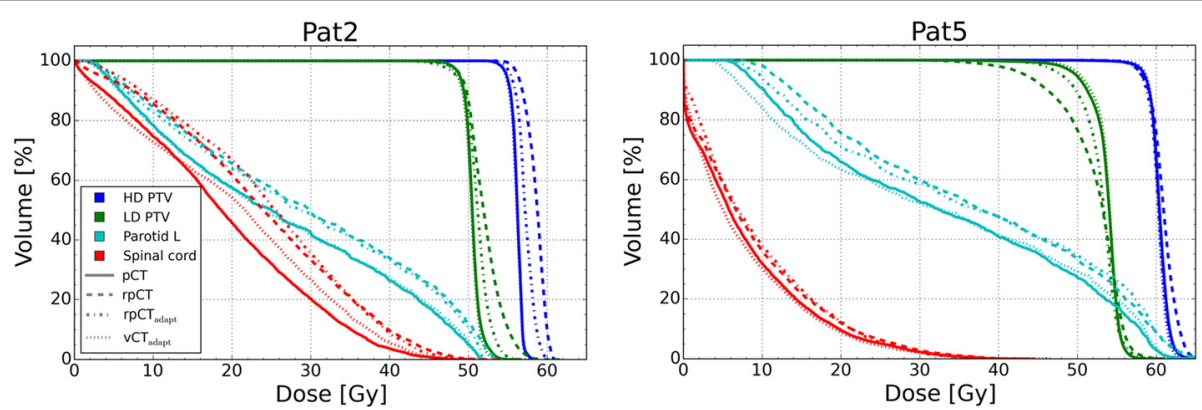

Fig. 2 DVH curves for Pat2 and Pat5 for the high dose PTV (HD PTV), low dose PTV (LD PTV), left (L) parotid gland and spinal cord PRV for the dose distributions of the initial plan on the $\mathrm{pCT}, \mathrm{rpCT}$ and the adapted plan on the rpCT (rpCT adapt) and $\mathrm{vCT}\left(\mathrm{v} C \mathrm{~T}_{\text {adapt }}\right)$. The LD PTV excludes the HD PTV volume expanded by a $5 \mathrm{~mm}$ margin 

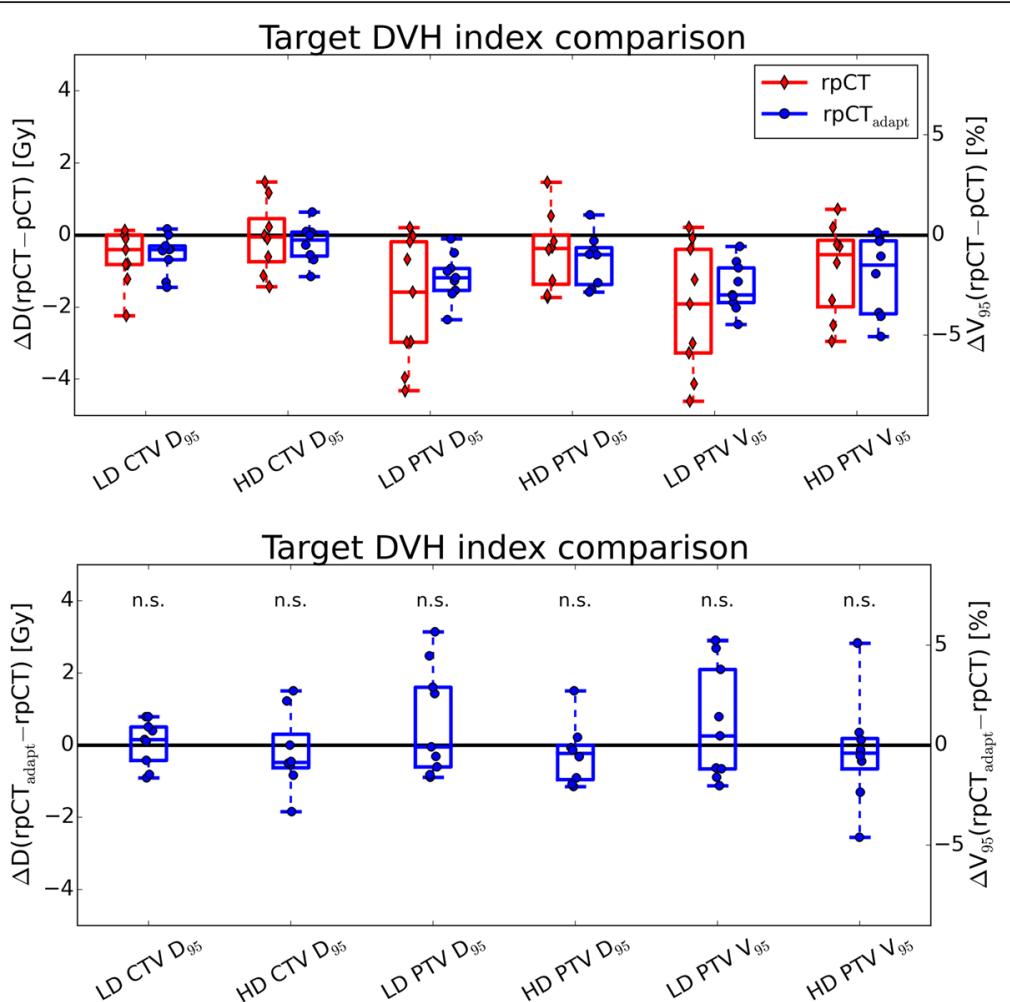

Fig. 3 Boxplots of target DVH index ( $D_{95}$ left vertical axis, $V_{95}$ right vertical axis) differences. The original (rpCT) and adapted plans ( $r p C T_{a d a p t}$ ) on the $\mathrm{rpCT}$ are compared to the original plan on the $\mathrm{pCT}$ (top panel) and to each other (bottom panel) for the 9 patients investigated in this study. Non-significant differences between original and adapted plan on the rpCT are indicated by 'n.s.'. All dose values refer to the total dose of the SIB treatment phase for ease of interpretation

showed that there were no significant differences in the high and low dose CTV and PTV $D_{95}$ and $V_{95}$ between the original and adapted plan on the $\operatorname{rpCT}(p>0.05)$. In terms of $D_{2}$, the adapted plans showed improved results for the high and low dose PTVs, as well as for the low dose CTV, with $p<0.05$. The effect on the high dose CTV was less pronounced ( $p=0.1$, see Fig. 4).

Figure 5 shows the data for OARs in similar fashion as Figs. 3 and 4. For OARs, the adapted plan showed no remarkable improvement with respect to the original plan

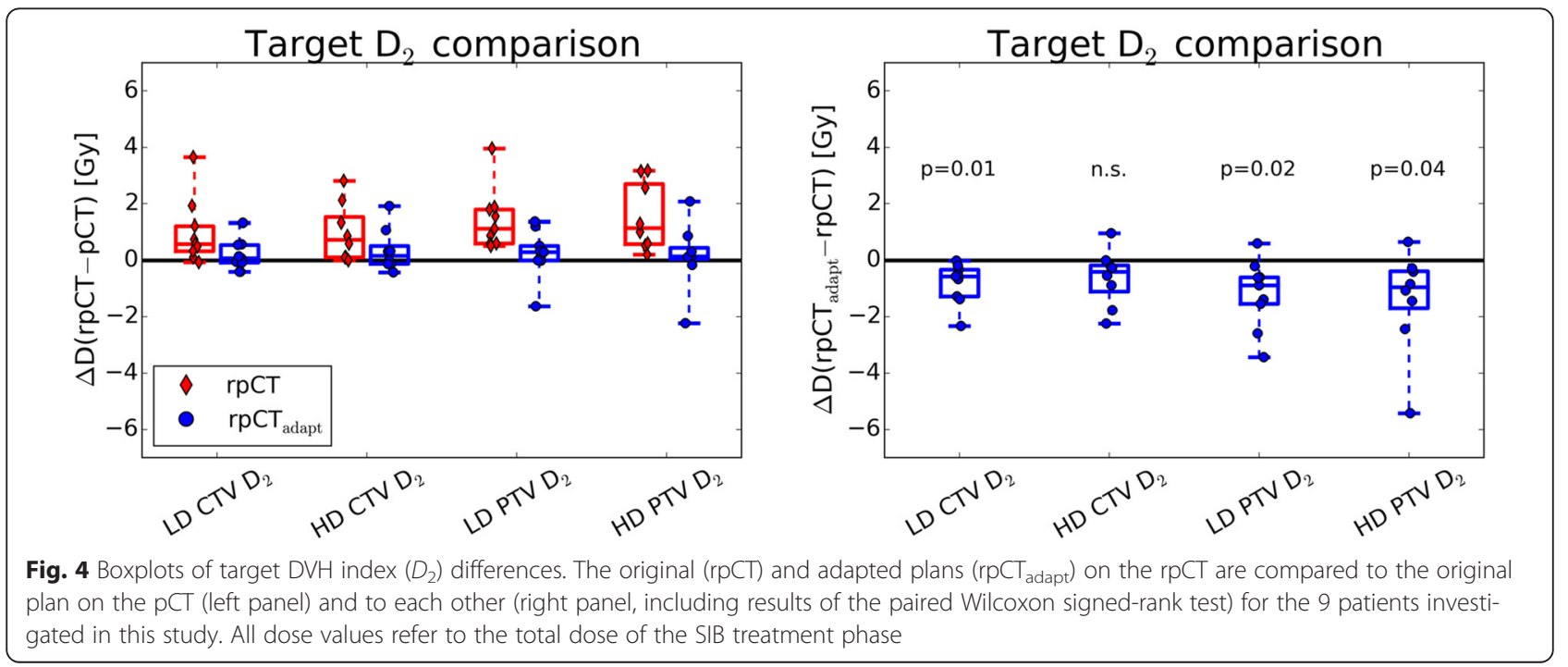



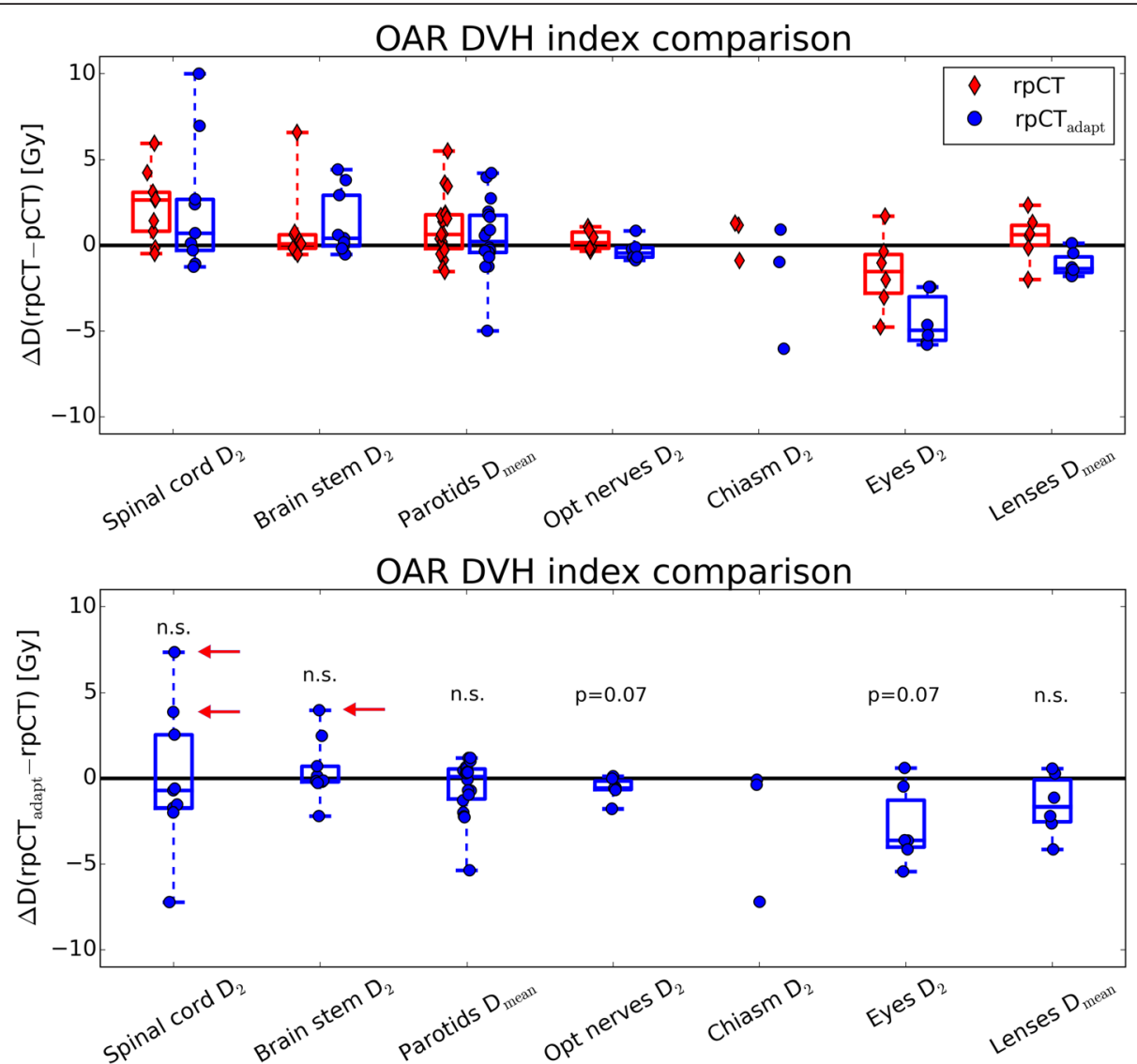

Fig. 5 Boxplots of OAR DVH index differences. The original $(r p C T)$ and adapted plans ( $r p C T_{\text {adapt }}$ ) on the rpCT are compared to the original plan on the PCT (top panel) and to each other (bottom panel, including results of the paired Wilcoxon signed-rank test) for the 9 patients investigated in this study. Left and right eyes, optical nerves and lenses were grouped together for Pat7-9, left and right parotid glands for all patients. All dose values refer to the total dose of the SIB treatment phase for ease of interpretation. Red arrows indicate the cases with most pronounced OAR dose increase from original to adapted plan (see Discussion section)

in the spinal cord, brain stem and parotid glands. Differences between the distributions were not significant. However, in two cases, spinal cord $D_{2}$ was increased by more than 3 Gy (28 Gy vs 24 Gy and 40 Gy vs 32 Gy) for the adapted plan with respect to the original plan. A considerable brain stem $D_{2}$ increase by 4 Gy (26 Gy vs $22 \mathrm{~Gy}$ ) was found in one case (see red arrows in Fig. 5). Still, for all cases the spinal cord and brain stem $D_{2}$ were well below the 53 Gy maximum dose objective used for treatment planning. For optical nerves $D_{2}$, chiasm $D_{2}$, eye $D_{2}$, and eye lenses $D_{\text {mean }}$ we observed a lower dose with $\mathrm{rpCT}_{\text {adapt }}$ at borderline significance ( $\mathrm{p}$ in the order of 0.1 ).

\section{Discussion}

In terms of the high and low dose PTVs and CTVs $D_{95}$ and $V_{95}$, the adapted plans yielded only minor differences compared to the original plans when evaluated on the rpCT, as observed on Fig. 3. The main improvement from the CBCT-based plan adaptation was the reduction of over-dosage in the target volumes, as indicated by the significantly reduced $D_{2}$ values (see Fig. 4 ). While overdosage in the GTV may not be of issue, the low dose PTV in H\&N cancer patients contains normal tissue where very high doses may not be desired.

For OARs, the adapted plans yielded improved results, i.e., lower DVH parameter, for optical nerves, chiasm, eyes and eye lenses with respect to the original plan. For the spinal cord, brain stem and parotid glands, no significant improvement by plan adaptation was found. With respect to the original planning scenario, the adapted plan evaluated on the rpCT shows a higher median OAR dose burden for parotid glands, brain stem and spinal cord (see Fig. 5, top row, blue boxplots). This indicates that the approach investigated here may not be sufficient to ensure optimal sparing of the OARs. However, following optimization of the adapted plan on the vCT, optimization targets for OARs, as well as all target structures, were always met within 1-2 Gy and corresponded to the original plan in terms of DVH parameter, 


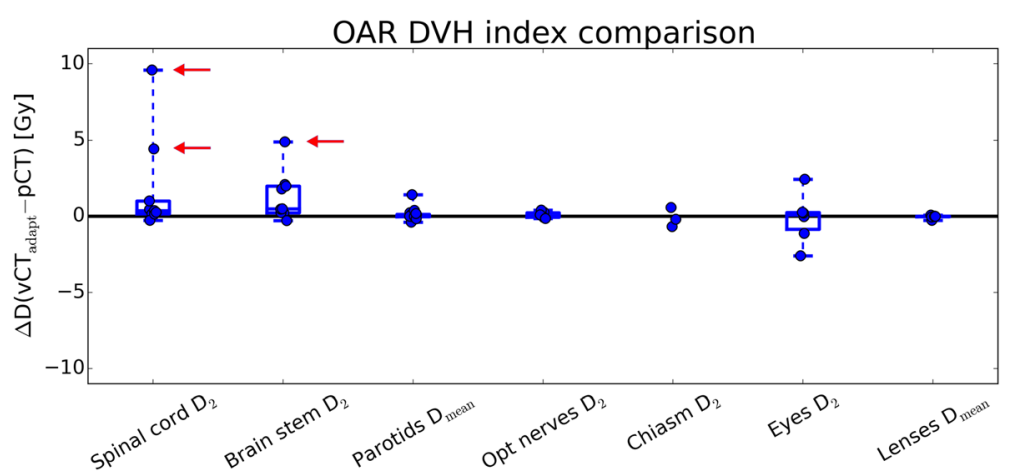

Fig. 6 Boxplots of OAR DVH index differences. The vCT optimized plan on the vCT is compared to the original planning scenario (original plan on $\mathrm{pCT}$ ) for the 9 patients investigated in this study. Red arrows indicate the cases with most pronounced OAR dose increase for the adapted plan. All dose values refer to the total dose of the SIB treatment phase

except from the above mentioned three cases with considerably increased spinal cord or brain stem $D_{2}$ (see Fig. 2 and Fig. 6). This is probably due to the fact that DVH values were far below the constraints used for plan optimization. In these cases, the dose to the spinal cord or brainstem, respectively, could easily be reduced to a level similar to the original planning scenario by using tighter constraints on the OAR dose during plan adaptation. Dose to other OARs or target structures were not compromised (see Additional file 1: Figure S1 for an exemplary case). This issue might be solved by automatic constraint adaptation as proposed in Breedveld et al. [30].

Remaining differences of the adapted plan recalculated on the $\mathrm{rpCT}$ with respect to the $\mathrm{vCT}$, e.g., in terms of the parotid glands $D_{\text {mean }}$ and the low dose PTV $D_{95}$ and $V_{95}$, can be due to several reasons: the IMPT plans may not be robust and slight differences in positioning, e.g., different neck tilts, between $\mathrm{rpCT}$ and $\mathrm{vCT}$ may be sufficient to negate optimal OAR sparing. Beyond, the DIR contours on the vCT could be inaccurate or there could be variations in physician contouring on the rpCT. The $\mathrm{pCT}, \mathrm{CBCT}$ and rpCT alignments were performed by focusing on a region of interest covering the $1^{\text {st }}$ to $6^{\text {th }}$ vertebra and manual adjustment of the registration yielded corrections of less than 1 voxel. However, by aligning the images using the spine there may be misalignment in other regions of the larger, low dose PTV. For a single patient we also employed a physician delineated $\mathrm{vCT}$ to generate the adapted plans and no marked differences were observed between that plan and the one optimized using the DIR delineation. Despite a comprehensive evaluation of the $\mathrm{vCT}$ approach by our group $[22,23]$, we cannot finally conclude on the basis of our patient cohort to what extent differences between $\mathrm{vCT}$ and rpCT (using the adapted plan) are related to the effects described above or to non-contour-related inaccuracies of the $\mathrm{vCT}$. To do so, a cohort of IMPT patients with little anatomical changes between repeated diagnostic CT imaging might be investigated to quantify the changes in DVH parameters solely due to re-positioning and re-contouring. In this respect, systematically employing automatic delineation tools may help reduce variability in delineation [31]. To preserve the DVH parameters obtained when optimizing the plan using the $\mathrm{vCT}$ it may also be necessary to employ robust optimization, or to use either different or automatically selected beam angles. A robust plan may provide worse parotid sparing initially but may mitigate the increased parotid dose observed on the rpCT.

The adapted plans were generated using a workflow where no human interaction was required in terms of contouring or optimization function adjustment. The procedure could thus be automated and started following the acquisition of the CBCT scan. Online adaptation is not realistic with this procedure given the computational time required for DIR, optimization and dose calculation (total of about $1 \mathrm{~h}$ ) and the method would thus serve to update the plan for the next fraction. This is the setting we investigated by making use of rpCTs acquired at a different time than the CBCT. We can assume that the anatomical and positioning differences between the rpCT acquisition and $\mathrm{CBCT}$ acquisition simulated the differences between two fractions. One key aspect of the $\mathrm{vCT}$ approach is that the patient anatomy and position correspond to the situation at the treatment couch, as opposed to the rpCT scan. Development of graphics processor unit based DIR and dose calculation may eventually allow the procedures described here to be performed online. If the $\mathrm{vCT}$ approach is not used to automatically adapt the treatment plan, it may still be used to evaluate the need for adaptation by monitoring DVH parameters, dose distributions directly or changes in the water equivalent thickness along beam paths. 


\section{Conclusion}

We have established an offline automatic procedure to generate an adapted IMPT plan on CBCT images. When evaluating the adapted plan on a physician delineated control rpCT we observed reduced over-dosage in the high and low dose PTV. OAR sparing was partially improved by the procedure, mainly for the optical system, but might demand for tighter constraints during plan adaptation. Despite potential limitations in the accuracy of the vCT approach, an improved quality of the adapted plans could be achieved. The implementation of the procedure in a clinical workflow would require evaluation of the adapted plans.

\section{Additional file}

Additional file 1: Figure S1. DVH curves for Pat3. The adapted plan (calculated on the $\mathrm{VCT}$ ) is compared to a re-optimized plan with a tighter constraint of 22 Gy for the maximum dose in the spinal cord. The original adapted plan is labelled vCT on the figure, while the plan with tighter constraints is labelled vCT OAR. Table S1. Patient target structure volumes from the $\mathrm{pCT}$ and $\mathrm{rpCT}$. All volumes in $\mathrm{cm}^{3}$. Patient neck volume changes were computed using the body contour in the neck region, excluding slices below the shoulders and above the jaw. This was not computed for patients with nasal cavity lesions. (PDF $279 \mathrm{~kb}$ )

\section{Abbreviations}

ART: adaptive radiotherapy; CBCT: cone beam computed tomography; CT: computed tomography; CTV: clinical target volume; DIR: deformable image registration; DVH: dose/volume histogram; GTV: gross tumor volume; H\&N: head and neck; HD: high dose; IMPT: intensity modulated proton therapy; IMRT: intensity modulated radiation therapy; L: left; LD: low dose; OAR: organ at risk; pCT: planning CT; PRV: planning risk volume; PTV: planning target volume; $\mathrm{ROI}$ : region of interest; rpCT: replanning $\mathrm{CT}$; SIB: simultaneous integrated boost; TPS: treatment planning system; vCT: virtual $C T$.

\section{Competing interest}

The authors declare that they have no competing interests.

\begin{abstract}
Authors' contribution
CK performed treatment planning, data analysis, generated figures and drafted parts of the manuscript. RN delineated planning and control $\mathrm{CT}$ scans. MR was responsible of the CBCT scanner related aspects of this study. UG performed delineation of planning and control $C T$ scans. $C T$ participated in the design of the study an in the data evaluation. CB and KP were responsible for the coordination of the study. GL performed treatment planning, deformable image registration and drafted parts of the manuscript. All authors read and approved the final manuscript.
\end{abstract}

\section{Acknowledgements}

This work was supported by the Federal Ministry of Education and Research of Germany (BMBF), Grant No. 01 IB13001 (SPARTA), and by the German Research Foundation (DFG) Cluster of Excellence Munich-Centre for Advanced Photonics (MAP).

The authors thank Guillaume Janssens and Jonathan Orban de Xivry for sharing the REGGUI toolkit containing the implementation of the Morphons algorithm. Helpful advice concerning IMPT treatment planning from Silvia Molinelli, Mario Ciocca, Martin Hillbrand, Daniel Köpl, Franz Joachim Kaizer is gratefully acknowledged. We thank Erik Traneus from RaySearch Laboratories for his support on the RayStation TPS.

Received: 16 June 2015 Accepted: 27 April 2016

Published online: 30 April 2016

\section{References}

1. Gupta T, Hotwani C, Kannan S, Master Z, Rangarajan V, Murthy V, et al. Prospective longitudinal assessment of parotid gland function using dynamic quantitative pertechnate scintigraphy and estimation of doseresponse relationship of parotid-sparing radiotherapy in head-neck cancers. Radiat Oncol. 2015:10:67. doi:10.1186/s13014-015-0371-2.

2. Studer $G$, Linsenmeier $C$, Riesterer $O$, Najafi Y, Brown M, Yousefi B, et al. Late term tolerance in head neck cancer patients irradiated in the IMRT era. Radiat Oncol. 2013;8:259. doi:10.1186/1748-717X-8-259.

3. Studer G, Rordorf T, Glanzmann C. Impact of tumor volume and systemic therapy on outcome in patients undergoing IMRT for large volume head neck cancer. Radiat Oncol. 2011;6:120. doi:10.1186/1748-717X-6-120.

4. Widesott L, Pierelli A, Fiorino C, Dell'oca I, Broggi S, Cattaneo GM, et al. Intensity-modulated proton therapy versus helical tomotherapy in nasopharynx cancer: planning comparison and NTCP evaluation. Int J Radiat Oncol Biol Phys. 2008;72(2):589-96. doi:10.1016/j.jirobp.2008.05.065.

5. Simone 2nd CB, Ly D, Dan TD, Ondos J, Ning H, Belard A, et al. Comparison of intensity-modulated radiotherapy, adaptive radiotherapy, proton radiotherapy, and adaptive proton radiotherapy for treatment of locally advanced head and neck cancer. Radiother Oncol. 2011;101(3):376-82. doi:10.1016/j.radonc.2011.05.028.

6. van de Water TA, Lomax AJ, Bij HP, de Jong ME, Schilstra C, Hug EB, et al. Potential benefits of scanned intensity-modulated proton therapy versus advanced photon therapy with regard to sparing of the salivary glands in oropharyngeal cancer. Int J Radiat Oncol Biol Phys. 2011;79(4):1216-24. doi:10.1016/j.jirobp.2010.05.012

7. van der Laan HP, van de Water TA, van Herpt HE, Christianen ME, Bijl HP, Korevaar EW, et al. The potential of intensity-modulated proton radiotherapy to reduce swallowing dysfunction in the treatment of head and neck cancer: A planning comparative study. Acta Oncol. 2013;52(3): 561-9. doi:10.3109/0284186X.2012.692885

8. Jakobi A, Bandurska-Luque A, Stützer K, Haase R, Löck S, Wack LJ, et al. Identification of Patient Benefit From Proton Therapy for Advanced Head and Neck Cancer Patients Based on Individual and Subgroup Normal Tissue Complication Probability Analysis. Int J Radiat Oncol Biol Phys. 2015;92(5): 1165-74. doi:10.1016/j.jijobp.2015.04.031.

9. Kraan AC, van de Water S, Teguh DN, Al-Mamgani A, Madden T, Kooy HM, et al. Dose uncertainties in IMPT for oropharyngeal cancer in the presence of anatomical, range, and setup errors. Int J Radiat Oncol Biol Phys. 2013; 87(5):888-96. doi:10.1016/j.ijrobp.2013.09.014.

10. Góra J, Kuess P, Stock M, Andrzejewski P, Knäusl B, Paskeviciute B, et al. ART for head and neck patients: On the difference between VMAT and IMPT. Acta Oncol. 2015;54(8):1166-74. doi:10.3109/0284186X.2015.1028590.

11. Müller BS, Duma MN, Kampfer S, Nill S, Oelfke U, Geinitz H, et al. Impact of interfractional changes in head and neck cancer patients on the delivered dose in intensity modulated radiotherapy with protons and photons. Phys Medica. 2015;31(3):266-72. doi:10.1016/j.ejmp.2015.02.007.

12. Park S, Cho M, Kim H. On-board CBCT/CBDT for Image-guided Proton Therapy: Initial Performance Evaluation. Int J Radiat Oncol Biol Phys. 2009; 75(Supplement Issue 3):S595-6. doi:10.1016/j.jirob.2009.07.1361.

13. Kurz C, Dedes G, Resch A, Reiner M, Ganswindt U, Nijhuis R, et al. Comparing cone-beam CT intensity correction methods for dose recalculation in adaptive intensity-modulated photon and proton therapy for head and neck cancer. Acta Oncol. 2015;54(9):1651-7. doi:10.3109/ 0284186X.2015.1061206.

14. Siewerdsen JH, Jaffray DA. Cone-beam computed tomography with a flatpanel imager: magnitude and effects of x-ray scatter. Med Phys. 2001;28(2): 220-31. doi: http://dx.doi.org/10.1118/1.1339879.

15. Fotina I, Hopfgartner J, Stock M, Steininger T, Lütgendorf-Caucig C, Georg D. Feasibility of CBCT-based dose calculation: comparative analysis of HU adjustment techniques. Radiother Oncol. 2012;104(2):249-56. doi:10.1016/j. radonc.2012.06.007.

16. Zhang T, Chi Y, Meldolesi E, Yan D. Automatic delineation of on-line headand-neck computed tomography images: toward on-line adaptive radiotherapy. Int J Radiat Oncol Biol Phys. 2007;68(2):522-30. doi:10.1016/j. ijrobp.2007.01.038.

17. Wu Q, Chi Y, Chen PY, Krauss DJ, Yan D, Martinez A. Adaptive replanning strategies accounting for shrinkage in head and neck IMRT. Int J Radiat Oncol Biol Phys. 2009;75(3):924-32. doi:10.1016/j.jijobp.2009.04.047.

18. Peroni M, Ciardo D, Spadea MF, Riboldi M, Comi S, Alterio D, et al. Automatic segmentation and online virtualCT in head-and-neck adaptive 
radiation therapy. Int J Radiat Oncol Biol Phys. 2012;84(3):e427-33. doi:10. 1016/.j.jirobp.2012.04.003.

19. Zhen X, Gu X, Yan H, Zhou L, Jia X, Jiang SB. CT to cone-beam CT deformable registration with simultaneous intensity correction. Phys Med Biol. 2012;57(21):6807-26. doi:10.1088/0031-9155/57/21/6807.

20. Zhen X, Yan H, Zhou L, Jia X, Jiang SB. Deformable image registration of CT and truncated cone-beam CT for adaptive radiation therapy. Phys Med Biol. 2013;58(22):7979-93. doi:10.1088/0031-9155/58/22/7979.

21. Veiga C, McClelland J, Moinuddin S, Lourenço A, Ricketts K, Annkah J, et al. Toward adaptive radiotherapy for head and neck patients: Feasibility study on using CT-to-CBCT deformable registration for "dose of the day" calculations. Med Phys. 2014;41(3):031703. doi:10.1118/1.4864240.

22. Landry G, Dedes G, Zöllner C, Handrack J, Janssens G. Orban de Xivry J et al. Phantom based evaluation of $\mathrm{CT}$ to $\mathrm{CBCT}$ image registration for proton therapy dose recalculation. Phys Med Biol. 2015;60(2):595-613. doi:10.1088/ 0031-9155/60/2/595.

23. Landry G, Nijhuis R, Dedes G, Handrack J, Thieke C, Janssens G, et al. Investigating $C T$ to $C B C T$ image registration for head and neck proton therapy as a tool for daily dose recalculation. Med Phys. 2015;42(3):1354-66. doi:10.1118/1.4908223.

24. Thomson DJ, Teo B-KK, Ong A, Ang KW, Kirk M, Ahn PH, et al. The Impact of Anatomic Change on Pencil Beam Scanning in the Treatment of Oropharynx Cancer. Int J Part Ther. 2015;2(2):394-403. doi:10.14338/JPT-15-00002.1 .

25. Veiga C, Alshaikhi J, Amos R, Lourenco AM, Modat M, Ourselin S, et al. Cone-beam computed tomography and deformable registration-based "dose of the day" calculations for adaptive proton therapy. Int J Part Ther. 2015;2(2):404-14. doi:10.14338/IJPT-14-00024.1.

26. Veiga C, Janssens G, Teng C-L, Baudier T, Hotoiu L, McClelland JR, et al. First clinical investigation of CBCT and deformable registration for adaptive proton therapy of lung cancer. Int J Radiat Oncol Biol Phys. 2016. doi: http://dx.doi.org/10.1016/j.jirobp.2016.01.055.

27. Hardcastle N, Tomé WA, Cannon DM, Brouwer CL, Wittendorp PW, Dogan $\mathrm{N}$, et al. A multi-institution evaluation of deformable image registration algorithms for automatic organ delineation in adaptive head and neck radiotherapy. Radiat Oncol. 2012;7:90. doi:10.1186/1748-717X-7-90.

28. Grégoire V, Eisbruch A, Hamoir M, Levendag P. Proposal for the delineation of the nodal CTV in the node-positive and the post-operative neck. Radiother Oncol. 2006;79(1):15-20. doi:10.1016/j.radonc.2006.03.009.

29. Janssens G, de Xivry JO, Fekkes S, Dekker A, Macq B, Lambin P, et al. Evaluation of nonrigid registration models for interfraction dose accumulation in radiotherapy. Med Phys. 2009;36(9):4268-76. doi: http://dx. doi.org/10.1118/1.3194750

30. Breedveld S, Storchi PR, Keijzer M, Heemink AW, Heijmen BJ. A novel approach to multi-criteria inverse planning for IMRT. Phys Med Biol. 2007; 52(20):6339-53. doi:10.1088/0031-9155/52/20/016.

31. Holliday EB, Frank SJ. Proton radiation therapy for head and neck cancer: a review of the clinical experience to date. Int J Radiat Oncol Biol Phys. 2014; 89(2):292-302. doi:10.1016/j.jijobp.2014.02.029.

\section{Submit your next manuscript to BioMed Central and we will help you at every step:}

- We accept pre-submission inquiries

- Our selector tool helps you to find the most relevant journal

- We provide round the clock customer support

- Convenient online submission

- Thorough peer review

- Inclusion in PubMed and all major indexing services

- Maximum visibility for your research

Submit your manuscript at www.biomedcentral.com/submit

Biomed Central 